\title{
Chinese guidelines for the assessment and provision of nutrition support therapy in critically ill children
}

\author{
Xue-Mei Zhu ${ }^{1}$ - Su-Yun Qian ${ }^{2} \cdot$ Guo-Ping Lu$^{1} \cdot$ Feng Xu ${ }^{3} \cdot$ Ying Wang ${ }^{4} \cdot$ Chun-Feng Liu ${ }^{5} \cdot$ Xiao-Xu Ren $^{6}$. \\ Yu-Cai Zhang ${ }^{7} \cdot$ Heng-Miao Gao $^{2} \cdot$ Tao Zhou $^{8} \cdot$ Hong-Xing Dang $^{3} \cdot$ Chong-Fan Zhang ${ }^{1,8} \cdot$ Yi-Min Zhu ${ }^{9}$
}

Received: 5 July 2018 / Accepted: 11 July 2018 / Published online: 28 August 2018

(c) Children's Hospital, Zhejiang University School of Medicine 2018

\begin{abstract}
Background This document represents the first evidence-based guidelines to describe best practices in nutrition therapy in critically ill children ( $>1$ month and $<18$ years), who are expected to require a length of stay more than 2 or 3 days in a Pediatric Intensive Care Unit admitting medical patients domain.

Methods A total of 25,673 articles were scanned for relevance. After careful review, 88 studies appeared to answer the preidentified questions for the guidelines. We used the grading of recommendations, assessment, development and evaluation criteria to adjust the evidence grade based on the quality of design and execution of each study.

Results The guidelines emphasise the importance of nutritional assessment, particularly the detection of malnourished patients. Indirect calorimetry (IC) is recommended to estimate energy expenditure and there is a creative value in energy expenditure, $50 \mathrm{kcal} / \mathrm{kg} / \mathrm{day}$ for children aged 1-8 years during acute phase if IC is unfeasible. Enteral nutrition (EN) and early enteral nutrition remain the preferred routes for nutrient delivery. A minimum protein intake of $1.5 \mathrm{~g} / \mathrm{kg} / \mathrm{day}$ is suggested for this patient population. The role of supplemental parenteral nutrition (PN) has been highlighted in patients with low nutritional risk, and a delayed approach appears to be beneficial in this group of patients. Immune-enhancing cannot be currently recommended neither in EN nor PN.

Conclusion Overall, the pediatric critically ill population is heterogeneous, and an individualized nutrition support with the aim of improving clinical outcomes is necessary and important.
\end{abstract}

Keywords Adolescents $\cdot$ Children $\cdot$ Critical illness $\cdot$ Enteral nutrition $\cdot$ Indirect calorimetry $\cdot$ Infant $\cdot$ Malnutrition · Nutrition assessment $\cdot$ Parenteral nutrition $\cdot$ Pediatric $\cdot$ Resting energy expenditure

Electronic supplementary material The online version of this article (https://doi.org/10.1007/s12519-018-0175-1) contains supplementary material, which is available to authorized users.

The Chinse version of the guidelines has been published in Chin J Evid Pediatr. 2018;13:1-29.

Su-Yun Qian

syqian1211@163.com

1 Children's Hospital, Fudan University, No. 399 Wanyuan Road, Minhang District, 201100 Shanghai, China

2 Beijing Children's Hospital, Capital Medical University, National Center for Children's Health, No. 56 Nan-li-shi Road, Xicheng District, 100045 Beijing, China

3 Children's Hospital of Chongqing Medical University, 136 Zhongshan No. 2 Road, Yu Zhong District, 400010 Chongqing, China

4 Shanghai Children's Medical Center, No. 1678, DongFang Road, 200127 Shanghai, China
5 Shengjing Hospital of China Medical University, No.36 Sanhao Road, 110004 Shenyang, China

6 The Capital Institute of Pediatrics, No. 2 Yabao Road, 100020 Beijing, China

7 Shanghai Children's Hospital, Shanghai Jiao Tong University, No. 355 Luding Road, 200062 Shanghai, China

8 Boai Hospital of Zhongshan Affiliated to Southern Medical University, 6 Chenggui Road, East District, 528400 Zhongshan, China

9 Hunan Provincial People's Hospital, No. 60 Jiefang West Road, 410005 Changsha, China 


\section{Introduction}

To promote and regulate the nutrition support therapy in critically ill children and to encourage corresponding academic research, a group of experts from the Emergency Group of Chinese Pediatrics Society, Pediatrics group of Chinese Emergency Society (both afflicted to Chinese Medical Association) and Clinical Guideline Production and Evaluation Center of Children's Hospital of Fudan University have examined all the available literatures and balanced potential benefits of nutrition practices against risks inherent with such therapy, and then developed the guidelines and authored this document. Since guidelines cannot account for every variation in circumstances, practitioners must always exercise professional judgment when applying these recommendations to individual patients. These Clinical Guidelines are intended to supplement, but not replace professional training and judgment in different pediatric intensive care units (PICU). These guidelines are not intended for specific diagnoses such as burn injuries, surgical related diseases (such as short bowel syndrome, SBS), diabetes, liver failure and so on. These guidelines are directed toward generalized patient, like any other management strategy in PICU, nutrition support therapy should be tailored to the individual patient.

The target of these guidelines is intended to be pediatric ( $>1$ month and $<18$ years) critically ill patient expected to require a length of stay (LOS) greater than 2 or 3 days in a PICU. These guidelines are intended for use by all healthcare providers involved in nutrition therapy of the critically ill children, primarily pediatric physicians, nurses, nutritionists, and pharmacists.

\section{Methods}

\section{Search strategy}

The methodologist performed a systematic search of the literature for relevant systematic reviews and individual studies using the following databases: China Biology Medicine Database (CBM), PubMed and EMBASE. Literatures on Chinese Medicine or combined Traditional Chinese Medicine-Western Medicine were excluded. The retrieval time was from the establishment of the database to Oct 31st, 2016. The research strategy of CBM, PubMed and EMBASE was constructed based on the topics related to nutrition assessment and support therapy in critically ill children including (1) nutritional risk screen, (2) nutrition assessment, (3) energy requirement, (4) principle of nutrient recommendation, (5) enteral nutrition and (6) parenteral nutrition. Each topic branched into 18 directions with corresponding research strategies. Research strategy of PubMed and EMBASE was "topic + direction" and research strategy for CBM was "topic" only.

\section{Literature extraction}

Preliminary screening excluded articles without abstracts, conference papers, editorials, lectures, experts' view, review (except for systematic review and meta-analysis), trials of newborn, adults and animals. After assessment of abstracts, selected articles were excluded if they were obviously irrelevant to the subtopics they belong to. We extracted relevant data from each eligible study into structured data tables. One panelist performed the data extraction and another panelist independently reviewed the extracted data. Discrepancies were resolved by discussion.

The inclusion criteria of studies of diagnostic accuracy are: clearly defined gold standard and diagnostic tools for evaluation; fourfold table data is available for calculation sensitivity and specificity. For cohort study and case-control study, odds ratio (OR) or standard error (SE) of exposures should be present in the article. For cross-sectional study and systemic report, exposure rate or incidence is required. For intervention studies including randomized controlled trial (RCT), non-RCT and before/after cohort study, measurements of outcome and actual sample size for analysis should be available.

\section{Results}

A total of 25,673 articles retrieved from the three databases were scanned for relevance. After careful review, 88 studies appeared to answer the pre- identified questions for this guideline.

\section{Question 1: What is the impact of nutritional status on outcomes in critically ill children?}

Recommendation Based on observational studies, we recommend $Z$ score for weight-for-height (WFH) and body mass index (BMI)-for-age (BFA) be used to screen patients at extremes of these values and to assess their detailed nutritional status. Malnutrition is associated with adverse clinical outcomes, including increased mortality (evidence level 1C-1D).

Rationale A retrospective study of prospectively collected data from the virtual PICU systems (VPS, LLC) database in America was conducted from January 1st, 2009, to March 31 st, 2013 [1]. There were 331,057 consecutive patient records identified, but only 127,607 (38.5\%) patients were included with height and weight recorded. They calculated $Z$ score for 
weight-for-age (WFA) according to the CDC/WHO growth curve and $Z$ score for WFH or BMI according to data with height indicator. And $Z$ score for WFA or for WFH/BMI separated into nine groups (Supplementary Fig. 1).

After adjusting for severity of illness with the Pediatric Index of Mortality 2 score (PIM2) and preexisting comorbidities, mortality had a $U$-shaped distribution when classified according to $Z$ score for WFA or $Z$ score for WFH/BMI. But adjusted mortality decreased into the lowest point with $z$ score at -0.5 to 1.49 on the WFA curve, while with $Z$ score at $-0.5-0.49$ on the WFH/BMI curve. It shows that when classifying patients using WFA without respect to height, the nadir of the mortality curve was shifted right, potentially falsely implying a benefit to mild obesity ( $Z$ score at $0.5-1.49$ ).

An observational study including two cohort studies conducted in $2009(n=524)$ and $2011(n=1245)$ [2] to determine the influence of admission anthropometry on clinical outcomes in mechanically ventilated children in the PICU. Data from 1622 eligible patients (aged 1 month-18 years) were analyzed. There were no significant differences in age, gender, $Z$ score for WFA, $Z$ score for HFA, $Z$ score for BMI, LOS, or 60-day mortality between the two cohorts. After adjusting for severity of illness and site, the odds of 60-day mortality were higher in underweight $(\mathrm{OR}=1.53 ; 95 \%$ $\mathrm{CI}=1.24-1.89 ; P<0.001)$ children but without difference between overweight $(\mathrm{OR}=1.44 ; 95 \% \mathrm{CI}=0.94-2.19)$ and obese $(\mathrm{OR}=1.55 ; 95 \% \mathrm{CI}=0.87-2.76)$ children when compared to normal weight children, respectively. Hazard ratios (HR) for hospital discharge were lower among underweight (HR $=0.71 ; 95 \% \mathrm{CI}=0.60-0.84 ; P<0.001)$ and obese $(\mathrm{HR}=0.82 ; 95 \% \mathrm{CI}=0.68-0.99 ; P=0.04)$ children. The $\mathrm{OR}$ of hospital-acquired infections were higher in underweight $(\mathrm{OR}=1.88 ; 95 \% \mathrm{CI}=1.18-3.01 ; P=0.008)$ and obese $(\mathrm{OR}=1.64 ; 95 \% \mathrm{CI}=1.33-2.03 ; P<0.001)$ children, while without difference in overweight children $(\mathrm{OR}=1.42 ; 95 \%$ $\mathrm{CI}=0.99-2.05)$. Nutritional status category was a significant contributor to the model predicting one or more ventilator free days (VFD) $\left(\chi^{2}=20.4 ; P<0.001\right)$, when controlling for age, gender, study year, diagnosis classification, illness severity, and PICU size. Underweight was associated with 1.3 (95\% CI -2.1 to $-0.6 ; P=0.001), 1.6(95 \% \mathrm{CI}-2.4$ to $-0.9 ; P<0.001)$ and $1.2(95 \% \mathrm{CI}-1.9$ to $-0.6, P<0.001)$ fewer VFDs, compared to normal weight, overweight, and obese children, respectively. Compared to the normal weight subjects, there were no significant differences in VFD among overweight and obese subjects.

\section{Question 2: How to screen and identify patients with malnutrition or at risk of nutritional deterioration in the PICU?}

Recommendation Based on observational studies we suggest that PYMS scale be used for screening and identifying patents at risk of malnutrition and that STRONGkids or STAMP scales can also be used (evidence level 2C-2D).

Rationale A study of the 247 children aimed to assess the diagnostic accuracy of different pediatric screening tools [3] showed that, compared to full dietetic assessments, the sensitivity of Screening Tool for the Assessment of Malnutrition in Paediatrics (STAMP), the Pediatric Subjective Global Nutritional Assessment (SGNA) and Pediatric Yorkhill Malnutrition Score (PYMS) were 81\% (95\% CI $=62-94)$, $15 \%(95 \% \mathrm{CI}=4-34)$ and $85 \%(95 \% \mathrm{CI}=66-96)$, respectively; and the specificity of the three tools were $78 \%$ (95\% $\mathrm{CI}=72-83), 100 \%(95 \% \mathrm{CI}=98-100)$ and $87 \%$ (95\% CI 82-91), respectively. When compared to PYMS, the sensitivity of STAMP and SGNA were $52.9 \%$ (95\% CI $=38-66)$ and $7.7 \%(95 \% \mathrm{CI}=2-19)$, with a specificity of $77.4 \%(95 \%$ $\mathrm{CI}=71-83)$ and $100 \%(95 \% \mathrm{CI}=98-100)$, respectively.

We used the Cochrane Collaboration Review Manager, version 5.0 to pool the results across individual studies and conducted a series of meta-analyses for the diagnostic accuracy of different nutritional risk screening scales. When WHO standard (WFH $Z$ value or HFA $Z$ value or BIM $Z$ value $<-2$ ) was taken as the gold standard for malnutrition, in diagnosing malnutrition in children with moderate and severe nutrition risk, the meta-analysis of four studies on diagnostic accuracy [4-7] $(n=1884)$ showed the sensitivity and specificity of STRONGkids were 0.64 (95\% $\left.\mathrm{CI}=0.57-0.71 ; I^{2}=92.9 \%\right)$ and $0.46(95 \% \mathrm{CI}=0.43-0.48$; $\left.I^{2}=93.2 \%\right)$, respectively. The meta-analysis of two studies $[4,7](n=1372)$ showed the sensitivity and specificity of PYMS were $0.74\left(95 \% \mathrm{CI}=0.65-0.81 I^{2}=97.0 \%\right)$ and 0.60 (95\% CI $0.58-0.63, I^{2}=99.0 \%$ ), which also showed that the sensitivity and specificity of STAMP were $0.69(95 \%$ $\left.\mathrm{CI}=0.60-0.77, I^{2}=97.0 \%\right)$ and $0.39(95 \% \mathrm{CI}=0.36-0.41$; $I^{2}=97.4 \%$ ). From higher to lower accuracy of different scales diagnosing children in malnutrition were PYMS, STRONGkids and STAMP sequentially. But it should be noted that there is a huge heterogeneity in the combined analyses.

\section{Question 3: What is the recommended energy requirement for critically ill children?}

Recommendation Based on observational cohort studies, we recommend that measured rest energy expenditure (MREE) by indirect calorimetry (IC) be used to determine energy requirements, and Schofield equation to estimate energy expenditure (EEE) if IC is unfeasible (evidence level 1, unrated). We suggest that $50 \mathrm{kcal} / \mathrm{kg} /$ day for children aged 1-8 years old or $880 \mathrm{kcal} /$ day for children aged $15-12$ years can be used as an estimated energy expenditure during the acute phase (evidence level 2, unrated).

Rationale We used the Cochrane Collaboration Review Manager, version 5.0 to pool the results across individual 
studies and Inverse Variance fixed effect model to calculate mean difference (MD) value for continuous outcomes with accompanying 95\% CI. Based on ten observational studies [8-17], an aggregated MREE value is about $49.4 \pm 19.5 \mathrm{kcal} /$ $\mathrm{kg} / \mathrm{day}(n=461)$ in critically ill children with a PICU stay from 1 to 198 days. EEEs derived from three different formulas have biases with MREE (Supplementary Fig. 2). It shows that the Schofield equation is the most closest to MREE and then the Talbot tables follows, which may underestimate daily energy expenditure, while the WHO equation tends to overestimate the daily energy expenditure.

Based on three studies [18-20], an aggregated MREE value is about $878.2 \pm 668.2 \mathrm{kcal} /$ day $(n=68)$ in critically ill children. EEEs derived from eleven equations and the biases between EEEs and MREE are shown in Supplementary Fig. 3. The Schofield equation also is most close to MREE.

It should be noted that: (1) the aggregated MREE are mostly derived from children with mechanical ventilation in PICU; (2) different researches use different units and statistics for MREE, but the "kcal/day" and "kcal/kg/day" are the two main units; (3) most studies have confirmed that MREE is more accurate than any other formulas in assessing energy expenditure, but it is not feasible in many situations with strict criterion. Technical criterias for inclusion of IC include an airleak less than $10 \%$ tidal volume a fractional inspired oxygen level no higher than 0.6 , patients being relatively stable, and absent or minimal bias flow from the ventilator. Given that children in PICU have severe illness and may change rapidly, MREE might not be conducted easily. So this guideline recommends $50 \mathrm{kcal} / \mathrm{kg} / \mathrm{day}$ for children aged 1-8 years or $880 \mathrm{kcal} /$ day for children aged 5-12 years to be considered as estimated energy expenditure in the early stage of the severe disease.

\section{Problem 4: What is the benefit of enteral nutrition (EN) in PICU population? Is early EN feasible in this group?}

Recommendation Based on observational studies, we recommend that initiating EN as soon as possible has a benefit in reducing mortality (evidence level 1C-1D). The adverse effects of EN when using vasoactive medications are mostly acceptable (evidence level 2D).

Rationale A historical cohort study in Brazil [21] evaluated the effect of parenteral nutrition (PN) and enteral nutrition (EN) on PICU mortality before and after the implementation of a nutrition support team (NST). After adjusting for the time of mechanical ventilation, age, PIM2 score and severe malnutrition $(Z$ score for WFA $\leq-3)$, et al., the risk of death was $83 \%$ lower in patients who received EN for about $50 \%$ of their $\operatorname{LOS}(\mathrm{OR}=0.17 ; 95 \% \mathrm{CI}=0.07-0.41)$.

A multicenter retrospective study conducted in 12 PICUs $(n=5105)$ in the United States [22], examined the association of early enteral nutrition (EEN) with mortality and morbidity in critically ill children. EEN was defined as the delivery of $25 \%$ of cumulative goal calories for the first 48 hours via the enteral route within the first 48 hours of PICU admission. After adjusting for propensity score, PIM2 score, age, and center, patients who received EEN were less likely to die in the first 30 days of PICU admission $(\mathrm{OR}=0.46 ; 95 \% \mathrm{CI}=0.29-0.74)$ or in the first 60 days of PICU admission $(\mathrm{OR}=0.51 ; 95 \% \mathrm{CI}=0.33-0.78)$ than those who did not receive EEN.

A retrospective chart review [23] of patients who received enteral nutrition during or within 24 hours of requiring continuous infusion of cardiovascular medications, such as dopamine, dobutamine, epinephrine, norepinephrine, or neo-synephrine, was conducted to evaluate the tolerance of $\mathrm{EN}$ in pediatric patients receiving cardiovascular medications. Fifty-five admissions (52 patients) met study criteria. Researchers found that four patients exhibited evidence of gastric intestinal (GI) bleeding, two patients had clinically insignificant heme-positive stools and the other two patients had underlying coagulopathy and more extensive bleeding. There were no other serious gastrointestinal complications. The study also showed that 16 of 55 patients (29\%) experienced feeding interruption for reasons related to perceived feeding intolerance (vomiting, abdominal distention, high gastric residuals, constipation, or diarrhea) and these GIrelated cases accounted for only 30 of 118 (25.4\%) reasons cited for interrupting feedings.

\section{Question 5: How should protein delivery goals be determined in critically ill children?}

Recommendation Supported by observational cohort studies, we recommend that physicians pay more attention to protein intake when EN conducted in critically ill children (evidence level $1 \mathrm{C}-1 \mathrm{D})$. We suggest a minimum protein intake of $1.5 \mathrm{~g} / \mathrm{kg} /$ day for this patient population (evidence level 2D).

Rationale A prospective, multicenter, cohort study [24] that included consecutive children (age 1 month-18 years) with mechanical ventilation for more than 48 hours in 59 PICUs from 15 countries, was conducted to examine the association of the adequacy of protein delivery with 60-day mortality. Researchers recorded the daily and cumulative mean adequacies of energy and protein delivery as a percentage of the prescribed daily goal during the PICU stay. The endpoint for nutritional data collection was 10 days or discharge from the PICU. After adjustment for PICU size, EN time, the duration in PICU, and disease severity, the adequacy of enteral protein intake was significantly associated with 60 -day mortality $(P<0.001)$, while the adequacy of enteral energy intake was not $(\mathrm{OR}=1.01 ; 95 \%$ $\mathrm{CI}=0.99-1.03)$. Compared to mean enteral protein intake adequacy $<20 \%$, intake adequacy in $20-60 \%$ and intake 
adequacy $>60 \%$ of the prescribed goal were associated with lower OR of $0.37(95 \% \mathrm{CI}=0.17-0.76)$ and 0.14 (95\% $\mathrm{CI}=0.04-0.52$ ) for 60-day mortality, respectively.

In a cohort study [25] sharing methods with another study included [22], they included patients from 31 PICUs of eight countries to describe the relationship between adequacy of energy and protein intake and clinical outcomes in mechanically ventilated children. After correcting nutritional assessment time, age, severity of illness, mechanical ventilation days, and gastrointestinal prokinetic agents, compared to mean energy intake adequacy $<33.3 \%$, intake adequacy in $33.3-66.7 \%$ and intake adequacy $>66.7 \%$ of the prescribed goal were associated with lower OR of $0.27(95 \%$ $\mathrm{CI}=0.11-0.76)$ and $0.14(95 \% \mathrm{CI}=0.03-0.61)$ for 60 -day mortality, respectively.

A Swiss single-center case-series report [26] was conducted to investigate how much protein and energy were needed to equilibrate nitrogen and energy balances on the basis of daily measurements of total urinary nitrogen (TUN), REE and protein and energy intakes in critically ill children who were predicted to have mechanical ventilation for 72 hours or more, taking the tracheal intubation or tracheal intubation for 15 days as the end point. TUN was measured by chemiluminescence, and REE was measured by indirect calorimetry. Based on 402 measurements performed in 74 children (median age 21 months), the mean TUN was high at $0.20 \mathrm{~g} / \mathrm{kg} /$ day $(95 \% \mathrm{CI}=0.20-0.22)$ and the REE was 55 $\mathrm{kcal} / \mathrm{kg} / \mathrm{day}(95 \% \mathrm{CI}=54-57)$. Nitrogen and energy balances were achieved with $1.5 \mathrm{~g} / \mathrm{kg} / \mathrm{day}(95 \% \mathrm{CI}=1.4-1.6)$ of protein and $58 \mathrm{kcal} / \mathrm{kg} /$ day $(95 \% \mathrm{CI}=53-63)$ of energy intake.

\section{Question 6: Are there any protein formulas we preferred?}

Recommendation Based on an observational study and a meta-analysis, it is not rare that hydrolyzed formula was given to critically ill children in clinical practice (evidence level 2D). No evidence shows that high-energy, high-protein formulas can shorten mechanical ventilation time or PICU days (evidence level 2C-2D).

Rationale In a single-center case-control study in Brazil [27], children admitted to ICU and receiving tube feeding for 48 hours were evaluated to identify the factors associated with the choice of peptide-based formulas in the first enteral nutrition prescription. Of 291 patients included, $85(29.2 \%)$ were given peptide-based formulas as the first nutrition prescription. The choice of peptide-based formulas preferred as the first enteral nutrition prescription was independently associated with malnutrition $(\mathrm{OR}=2.94$; 95\% CI $=1.60-5.39 ; P<0.01)$, fasting period $>2$ days $(\mathrm{OR}=3.46 ; 95 \% \mathrm{CI}=1.93-6.20 ; P<0.01)$, and use of adrenergic drugs $(\mathrm{OR}=2.32 ; 95 \% \mathrm{CI}=1.24-4.31 ; P<0.01)$. We conducted a meta-analysis $(n=36)$ of two RCTs [28, 29] of the different nitrogen balance test methods published in the same center in 2009 and 2011. The mechanical ventilation time ( $\mathrm{MD}=1.66$ days; $95 \% \mathrm{CI}=0.16-3.15)$ and $\mathrm{PICU}$ stay ( $\mathrm{MD}=2.30$ days, $95 \% \mathrm{CI} 0.49-4.11)$ were prolonged in the higher energy and higher protein formula group $(n=16)$ than in the standard formula group $(n=20)$.

\section{Question 7: Route of enteral nutrition: What is the best site and optimum method for EN delivery?}

Recommendation Based on RCT studies, we recommend that continuous enteral feeding or the transpylorus enteral feeding are more likely to achieve target daily energy goal compared with intermittent feeding or transgastric feeding, respectively (evidence level 1D); on the basis of available evidence, both of the treatments above cannot reduce the incidence of aspiration or ventilator-associated pneumonia (VAP) (evidence level 1C-1D); EN can be performed sooner in transgastric enteral feeding than transpylorus enteral feeding (evidence level 2C).

Rationale The meta-analysis of two RCTs [30, 31] found no significant difference in the risk of aspiration between gastric and small bowel feeding groups ( $R R=0.79 ; 95 \%$ $\mathrm{CI}=0.54-1.17)$. In a RCT study [31], intubated patients (age $<18$ years) were randomized to receive either nasogastric $(n=27)$ or postpyloric $(n=17)$ enteral feeding. The waiting time for beginning feedings in the postpyloric enteral feeding group was longer than in the nasogastric group [24 hours (95\% CI $=18-24)$ vs. 6 hours (95\% CI $=6-12)$; $P<0.05)]$.

In a single center RCT in Turkey [32], forty pediatric patients requiring mechanical ventilation for more than 48 hours were randomised and divided into two groups of 20 , one group for nasoduodenal (ND) feeding and the other for nasogastric (NG) feeding. Patients were assessed for the development of VAP using the clinical pulmonary infection score and CDC criteria. There was no significant difference in age, gender, diagnosis, complications, nutrition time, stress ulcer nor the mortality $(P>0.05)$ between the two groups. No statistically significant difference in the incidence of VAP was observed between the ND- and NG-fed patients $(\mathrm{RR}=2.0 ; 95 \% \mathrm{CI}=0.41-9.71)$.

\section{Question 8: Factors affecting EN implementation}

Recommendation According to the observational studies, EN interruption is very common and mechanical ventilation is an independent predictor of EN interruption (evidence level 2D); non-invasive ventilation, invasive ventilation, disease severity, fluid limitation, clinical procedures, and gastrointestinal complications all increase the risk of EN delay (evidence level 2C-2D); Acute renal failure, together with 
continuous renal replacement therapy (CRRT) affect protein and energy intake in EN (evidence level 2D).

Rationale In a single center case-control study [33] of 80 patients receiving EN for 24 hours or more during their PICU stay, EN interruption was defined as an episode of stoppage or delay of EN for a period longer than 30 minutes. Causes of EN interruption include endotracheal tube extubation or intubation, diagnostic tests or procedures in the radiology suite, other procedures at the bedside, surgical procedure in the operating room, intolerance to EN and mechanical issues related to feeding tubes. 58\% (51/88) episodes of EN interruptions were deemed as avoidable in $15 / 80$ patients. Multivariate analysis identified EN time and mechanical ventilation as the most significant independent predictors of EN interruption, and that each additional day of EN was associated with an increasing odds of $39 \% \mathrm{EN}$ interruption $(\mathrm{OR}=1.39,95 \% \mathrm{CI}=1.20-1.60)$.

In a multicenter case-control study (six PICU) in the United States, [34] patients were divided into two groups based on the timing of EN initiation. Patients with EN started $<48$ hours after PICU admission were in the early EN group $(n=356)$ and $\geq 48$ hours were in the delayed EN group $(n=88)$. Risk factors associated with delayed EN were: non-invasive $(\mathrm{OR}=3.37 ; 95 \% \mathrm{CI}=1.69-6.72)$ and invasive positive pressure ventilation $(\mathrm{OR}=2.06$; $95 \% \mathrm{CI}=1.15-3.69$ ), severity of illness (OR for every 0.1 increase in PIM2: 1.39; 95\% CI $=1.14-1.71$ ), procedures $(\mathrm{OR}=3.33 ; 95 \% \mathrm{CI}=1.67-6.64)$ and gastrointestinal disturbances $(\mathrm{OR}=2.05 ; 95 \% \mathrm{CI}=1.14-3.68)$. Delayed $\mathrm{EN}$ was associated with failure to reach full EN while in the PICU $(\mathrm{OR}=4.09 ; 95 \% \mathrm{CI}=1.97-8.53)$.

A single center historical cohort study [35] enrolled two groups of 41 consecutive patients admitted to PICU from 1st Jan to 31st August in 2000 and 2003, respectively, aiming to determine the impact of nutrition support team (NST) on energy intake. Multivariate analysis indicates that a pediatric risk of mortality (PRISM) score $>10(\mathrm{OR}=0.58$; $95 \% \mathrm{CI}=0.44-0.77)$, a fluid restriction $(\mathrm{OR}=0.51$; $95 \% \mathrm{CI}=0.37-0.71$ ), and a weight for age $<3$ rd centile $(\mathrm{OR}=0.54 ; 95 \% \mathrm{CI}=0.41-0.72)$ are associated with a delay to achieve a sustained optimal caloric intake (SOCI).

\section{Question 9: Clinical outcomes of immune-enhancing diet in EN}

Recommendation According to the meta-analyses conducted by experts from Chinese Medical Association, we do not recommend the use of immunonutrition in critically ill children (evidence level 1C-1D).

Rationale The current EN immunoenhancing agents mainly include L-glutamic acid, L-arginine, fish oil ( $\omega 3$ polyunsaturated fatty acids) and dietary fiber. (1) The metaanalysis of six RCTs [36-41] found no statistical difference in survival rate between immune-enhancing group $(n=279)$ and non immune-enhancing group $(n=271)(\mathrm{RR}=0.96 ; 95 \%$ $\mathrm{CI}=0.91-1.00)$ (Supplementary Fig. 4); (2) a meta-analysis of four RCTs [36, 38, 39, 42] found that the length of hospital stay had not reduced significantly in immune-enhancing group $(n=69)$ than in non immune-enhancing group $(n=66)$ $(\mathrm{MD}=-0.46$ days; $95 \% \mathrm{CI}=-1.64-0.73$ ) (Supplementary Fig. 5); (3) a meta-analysis of two RCTs [38, 41] indicates that, compared to the non immune-enhancing group $(n=72)$, the length of PICU stay of immune-enhancing group $(n=74)$ reduced by 1.5 days $(\mathrm{MD}=-1.48 ; 95 \% \mathrm{CI}-2.44$ to -0.51$)$ (Supplementary Fig. 6); (4) a meta-analysis of three RCTs $[36,38,39]$ indicated that, compared with the non immuneenhancing group $(n=47)$, the duration of mechanical ventilation in the immune-enhancing group $(n=49)$ was prolonged by 2 days $(\mathrm{MD}=2.20 ; 95 \% \mathrm{CI}=1.31-3.09$ ) (Supplementary Fig. 7); (5) a meta-analysis of five RCTs [36-40] found no significant difference in the rate of hospital acquired infection ( $\mathrm{RR}=1.01 ; 95 \% \mathrm{CI}=0.82-1.25)$ between the immune-enhancing group $(n=223)$ and non immuneenhancing group $(n=216)$ (Supplementary Fig. 8$)$; (6) a meta-analysis of two RCTs [38, 40] showed that no significant difference in the incidence of adverse events $(R R=1.03$; 95\% $\mathrm{CI}=0.96-1.12$ ) between the immune-enhancing group $(n=162)$ and non immune-enhancing group $(n=151)$ (Supplementary Fig. 9); (7) a single RCT [42] demonstrated that, compared to the non immune-enhancing group $(n=19)$, the cost in immune-enhancing group $(n=20)$ reduced by 14,000 RMB (MD $=-1.35 ; 95 \% \mathrm{CI}-1.93$ to -0.77$)$.

\section{Question 10: EN complications}

Recommendation EN complications include but not limited to aspiration and aspiration pneumonia; nausea, vomiting, abdominal distension, diarrhea, constipation; bowel necrosis, mucosal atrophy; hyperglycemia and hypoglycemia, electrolyte imbalance, hypercapnia and refeeding syndrome (evidence level 2D).

Rationale The literature on reporting complications of EN is not rare. It is most common in secondary outcome indicators or in some case-series reports. Due to the uncertain volume of relevant articles and to the difficulty in extracting information by reviewing all the titles or abstracts, our recommendation is based on authoritative textbooks in the field [43].

\section{Question 11: Supplemental parenteral nutrition (PN)}

Recommendations Based on a RCT study and expert opinion for patients with low nutritional risk who do not receive enough energy through EN, we recommend that supplementary PN be initiated 1 week later, which do not increase 
mortality, but reduce the rates of new infections and shorten LOS of hospitalization, while increase the risk of hypoglycemia (evidence level 1A-1B).

Rationale In a multicenter RCT [44], children (from mature neonatal to children 17 years old) who were admitted to PICU and expected to stay for 24 hours or more, with a STRONGkids score of 2 or more were eligible for inclusion. The exclusion criteria included: no need of nutritional support; transfer from another PICU; readmission to PICU; TPN; metabolic disease or tumor. All patients enrolled were divided into two groups: early PN group (PN was initiated within 24 hours after admission to PICU, $n=723$ ) and late PN group (PN was withheld up to the morning of day 8 in the PICU, $n=717$ ). Eligible patients were randomly assigned to one of the two groups in 1:1 ration using a central computerized randomization system. In both groups, EN was initiated early. The primary outcomes were new infections acquired during the ICU stay, the duration of ICU and the proportion of patients requiring $\geq 8$ days in PICU; the secondary outcomes were mortality, duration of MV support, the incidence of hypoglycemia in the first 7 days in PICU. There were no significant differences in the characteristics of patients in both groups at baseline. After adjustment for center, age, PELOD score and STRONGkids score, the percentage of patients with a new infection was 10.7\% in the late PN group, as compared with $18.5 \%$ in early PN group (adjusted $\mathrm{OR}=0.48 ; 95 \% \mathrm{CI}=0.35-0.66$ ). As compared with $9.2 \pm 0.8$ days in the group receiving early PN, the mean $( \pm \mathrm{SE})$ duration of ICU stay was $6.5 \pm 0.4$ days in the group receiving late $\mathrm{PN}$, in whom the stay of PICU reduced by approximately 1 day $(\mathrm{MD}=-1.23 ; 95 \% \mathrm{CI}$ -1.11 to -1.37$)$. When compared to early PN, later PN group was associated with one day shorter for mechanical ventilatory support $(\mathrm{MD}=-1.19 ; 95 \% \mathrm{CI}-1.07$ to -1.32$)$, a shorter duration of hospital stay $(P=0.001)$, a decreased proportion of $26 \%$ within patients requiring $\geq 8$ days in PICU (RR $=0.74 ; 95 \% \mathrm{CI}=0.62-0.89)$, but an increased incidence of $87 \%$ for hypoglycemia (glucose $<2.2 \mathrm{mmol} / \mathrm{L}$ ) $(\mathrm{RR}=1.87 ; 95 \% \mathrm{CI}=1.26-2.79)$. There were no significant differences in the mortality within 8 days of admission to PICU ( $\mathrm{HR}=0.73 ; 95 \% \mathrm{CI}=0.34-1.51)$, during stay in PICU ( $\mathrm{HR}=0.73 ; 95 \% \mathrm{CI}=0.42-1.28)$, during hospital stay $(\mathrm{HR}=0.72 ; 95 \% \mathrm{CI}=0.43-1.19)$ and within 90 days after enrollment $(\mathrm{HR}=0.64 ; 95 \% \mathrm{CI}=0.39-1.05)$. But it should be noted that the population of the study was characterized by (1) the low nutritional risk on admission ( $>90 \%$ patients had a medium STRONGkids risk level); (2) received approximately $30 \mathrm{kcal} \mathrm{kg} / \mathrm{day}$ EN within 4 days after PICU admission; (3) up to $70 \%$ of the patients discharged from PICU within 8 days; (4) $50 \%$ of the population were surgical patients. Therefore, in patients who are severely malnourished or at high risk of nutritional deterioration, the initiation of PN should be based on the individual situation, and can be supplemented in the first week if they are unable to advance past low volumes of EN.

\section{Problem 12: Immune-enhancing in PN}

Recommendation Based on one RCT study, we do not suggest the use of glutamine in routine PN (evidence level 2 B-D).

Rationale A single center RCT [45] was conducted in PICU patients (aged from 1-month to 14-year old) requiring PN $>5$ days in Spain. Patients were allocated either to the experimental group (SPN with Gln supplementation) or to the control group (SPN) according to their assigned random number. The outcomes were occurrence of nosocomial infection, duration of inotropes and mechanical ventilation, length of stay in PICU and in-hospital mortality. Patients were randomly assigned to standard parenteral nutrition (SPN, 49 subjects) or standard parenteral nutrition with glutamine supplementation (SPN + Gln, 49 subjects, in which one patient was excluded because of death and two patients were excluded due to lack of baseline clinical data). ITT analysis was not conducted. No significant differences were found in demographic, clinical characteristics and nutritional support including caloric, proteins and lipids intake between the two groups. There were no statistical differences in nosocomial infections $(\mathrm{RR}=1.4 ; 95 \% \mathrm{CI}=0.76-2.51)$, mortality $(\mathrm{RR}=1.5 ; 95 \% \mathrm{CI}=0.26-8.59)$, duration of mechanical ventilation (135.8 vs. 134.0 hours, $P=0.98$ ), length of PICU stay (10.4 vs. 14.3 days, $P=0.062$ ), length of hospitalization (14.3 vs. 16.7 days, $P=0.098$ ) between the $\mathrm{PN}+\mathrm{Gln}$ group and PN group.

\section{Question 13: PN complications}

Recommendation The complications of PN [43] include but not limited to hyperglycemia and hyperosmolar hyperglycemic nonketotic coma, hypoglycemia, hyperlipidemia, fat over load syndrome, hyperammonemia, cholestatic jaundice, liver dysfunction, deficiency or overload of mineral and trace elements; prolonged TNP might induce mucosal atrophy, decreased secretion of intestinal glands, increased bile viscosity and loss of appetite (evidence level 2D). Postmortem revealed a possible association between TPN and lung fibrosis (evidence level 2D).

Rationale A single center case-control study in Brazil [46] was designed to describe lung injuries in autopsied pediatric patients subjected or not to total parenteral nutrition (TPN). A total of 301 children suffered fatal outcome from 1998 to 2001, and 114 of these cases permission were granted by their parents for autopsy. The 114 patients were divided in two groups: 50 were treated with TPN containing lipid emulsion and 64 did not receive TPN. The duration of hospital stay $(P=0.000)$, prematurity $(P=0.008)$ and 
treatment with blood products $(P=0.009)$ were all higher in the group treated with TPN. Chi square comparisons showed that diffuse alveolar injury $(P=0.022)$, pulmonary fibrosis $(P=0.019)$, pneumocyte hyperplasia $(P=0.004)$, micro thromboembolism $(P=0.047)$ and thrombophlebitis $(P=0.033)$ all exhibited a significant relationship with TPN. However, a multivariate analysis by logistic regression, taking into account prematurity and duration of hospital stay, demonstrated that TPN was an independent factor with respect of pulmonary fibrosis $(\mathrm{OR}=5.76 ; 95 \%$ $\mathrm{CI}=1.30-25.48$ ).

\section{Question 14: Adequacy of nutrient support}

Recommendation According to observational studies, under delivery of energy and protein in PICU requires more attention (evidence level 1D).

Rationale A single center retrospective chart review in the US [47] included 240 patients (aged from 36 weeks to 18 years) admitted to PICU for longer than 72 hours, in whom readmission patients were excluded. Basal metabolic rate (BMR) and protein requirements were estimated by Schofield equation and the ASPEN recommendations, respectively. During the first 8 days of PICU stay, the actual energy intake for all patient-days was an average of $(75.7 \pm 56.7) \%$ of BMR and was significantly lower than BMR $(P<0.001)$; the actual protein intake for all patientdays met an average of $(40.4 \pm 44.2) \%$ of protein requirements and was significantly lower than the ASPEN recommendations $(P<0.001)$.

In a single center case-series report in Brazil [48], PICU patients who received EN for at least 48 hours were enrolled, and patients with chronic diarrhea, abdominal surgery, development of peritonitis, or gastrointestinal hemorrhage were excluded. Prescribed calories and delivered calories were checked daily and compared with BMR (from WHO equations). Results demonstrated that under prescription, defined as a ratio of prescribed: required calories $<90 \%$, occurred in $42.4 \%$ (157/370) of the EN days, and underfeeding, defined as the ratio of delivered: required calories $<90 \%$ of the predicted BMR, presented in $55.7 \%(206 / 370)$ of the EN days.

\section{Question 15: Nutrition support team (NST) and feeding protocol}

Recommendation Based on observational cohort studies, we suggest that NST will be beneficial to PICU population (evidence level 1B-1D) and that a feeding protocol can help to achieve target energy goal earlier and reduce avoidable EN interruptions (evidence level 1D).
Rationale A multicenter $(n=5)$ case-control study [49] in 2013 recruited 1349 children consecutively admitted to PICU. Patients were allocated to caloric recorded group $(n=644)$ and no caloric recorded group $(n=705)$ based on whether a caloric requirement is estimated and documented in the medical records within $48 \mathrm{~h}$ of PICU admission. Nutritional intake data were gathered on the first 4 days of PICU admission. In the caloric recorded group, caloric requirement was determined $95.6 \%$ by registered dietitians. The results showed that: (1) patients with a documented caloric requirement recorded had higher total daily energy intake (reported as a percentage of the estimated daily REE) by EN within the first 4 days, ranging from 9 to $20 \%$; (2) patients with a documented caloric requirement had higher total daily energy intake by EN and PN combined on the first 4 days of PICU stay, ranging from 6 to $23 \%$; (3) patients with a documented caloric requirement were $1.5-1.8$ times more likely to receive EN than those without a documented caloric requirement, on each of the first 4 days after admission.

A single center observational study [50] of sustained quality improvement in Britain retrospectively collected the EN data of 355 patients over the past 10 years. The number of patients enrolled in each audit respectively was 83 in 1994-1995, 72 in 1997-1998, 100 in 2001 and 100 in 2004-2005. There were no statistically significant difference in age and gender among the four groups. The researchers found that: (1) time taken to initiate nutritional support (h) in four periods were: 15 (2-74), 8 (1-72), 5.5 (1-43) and 4.5 (1-38), respectively; (2) percentage of patient receiving at least $50 \%$ of the estimated average requirement by day 3 in four periods were: $15 \%(12 / 83)$, 26\% (19/72), 58\% (58/100) and 59\% (59/100), respectively; (3) percentage of patient receiving at least $70 \%$ of the estimated average requirement by day 3 in four periods were: $6 \%(5 / 83), 10 \%$ (7/72), 35\% (35/100) and $21 \%(21 / 100)$, respectively. Significant differences were observed in the three variates mentioned above between the 1994-1995 and 2005 groups $(P=0.001)$.

In a single center cohort in the US [51], the investigators built up NST and instituted a feeding protocol, and took a 2-month period to become acclimated to use this new protocol. 93 medical patients who met entry criteria were enrolled in 2002, with a comparison group of 91 consecutive patients in 2000. Compared to the 2000 group, the 2002 group reached goal nutrition in a shorter time (18.5 vs. 57.8 hours, $P<0.0001$ ), and were less likely to experience diarrhea $(\mathrm{RR}=0.18 ; 95 \% \mathrm{CI}=0.04-0.78)$. There were no significant difference in the length of hospitalization $(\mathrm{MD}=1.8$ days; $95 \% \mathrm{CI}=-3.51$ to 7.11$)$, length of PICU stay (MD $=-0.40$ days; $95 \% \mathrm{CI}=-3.28$ to 2.48 ), and incidence of vomiting ( $\mathrm{RR}=0.54 ; 95 \%$ $\mathrm{CI}=0.27-1.11)$ between the two groups. 


\section{Conclusions}

In this brief document, we have provided guidelines for some important steps in the assessment and provision of optimal nutrition to the critically ill children. We have answered the pre-identified questions for this guideline based on systematic search strategy and fully evaluating the quality of each evidence. The guidelines emphasize the importance of nutritional assessment, particularly the detection of malnourished patients. IC is recommended to estimate energy expenditure and there is a creative value in energy expenditure, $50 \mathrm{kcal} / \mathrm{kg} /$ day for children aged 1-8 years old during acute phase if IC is unfeasible. Enteral nutrition and early enteral nutrition remain the preferred routes for nutrient delivery. A minimum protein intake of $1.5 \mathrm{~g} / \mathrm{kg} /$ day is suggested for this patient population. The role of supplemental parenteral nutrition has been highlighted in patients with low nutritional risk, and a delayed approach appears to be beneficial in this group of patients.

Immune-enhancing cannot be currently recommended neither in EN nor PN. We suggest NST and feeding protocols used to achieve target energy goal earlier and reduce complications during nutrition therapy. Overall, the pediatric critically ill population is heterogeneous, and an individualized nutrition support with the aim of improving clinical outcomes is necessary and important. We hope that we have already captured all the relevant studies and abstracted the data in detail, but we are aware that some of these questions and several other questions remain unanswered. A majority of the recommendations in these guidelines are driven by low or very low-level evidence. It is meaningful but a challenge to convert all the recommendations to clinical practice. Therefore, multidisciplinary collaborative efforts must be devoted to prioritize and highlight the individual nutritional needs of the critically ill children in the complex PICU environments.

Author contributions All authors contributed equally to this paper.

Funding This study was funded in total by internal funds from the Emergency group of Chinese Pediatrics Society and Pediatrics Group of Chinese Emergency Society, both of which are affiliated to the Chinese Medical Association.

\section{Compliance with ethical standards}

Ethical approval Not required for this Guideline.

Conflict of interest No financial interests have been received directly or indirectly related to the subject of this article. The guidelines are intended for general information only, and do not replace professional medical care and physician advice, which always should be sought for any medical condition.

\section{References}

1. Ross PA, Newth CJ, Leung D, Wetzel RC, Khemani RG. Obesity and mortality risk in critically ill children. Pediatrics. 2016;137:e20152035.

2. Bechard LJ, Duggan C, Touger-Decker R, Parrott JS, RothpletzPuglia P, Byham-Gray L, et al. Nutritional status based on body mass index is associated with morbidity and mortality in mechanically ventilated critically ill children in the PICU. Crit Care Med. 2016;44:1530-7.

3. Gerasimidis K, Keane O, Macleod I, Flynn DM, Wright CM. A four stage evaluation of the Paediatric Yorkhill Malnutrition Score in a tertiary paediatric hospital and a district general hospital. $\mathrm{Br}$ J Nutr. 2010;104:751-6.

4. Chourdakis M, Hecht C, Gerasimidis K, Joosten KF, Karagiozoglou-Lampoudi T, Koetse HA, et al. Malnutrition risk in hospitalized children: use of 3 screening tools in a large European population. Am J Clin Nutr. 2016;103:1301-10.

5. Spagnuolo MI, Liguoro I, Chiatto F, Mambretti D, Guarino A. Application of a score system to evaluate the risk of malnutrition in a multiple hospital setting. Ital J Pediatr. 2013;39:81.

6. Huysentruyt K, Alliet P, Muyshont L, Rossignol R, Devreker $\mathrm{T}$, Bontems P, et al. The STRONG(kids) nutritional screening tool in hospitalized children: a validation study. Nutrition. 2013;29:1356-61.

7. Moeeni V, Walls T, Day AS. Assessment of nutritional status and nutritional risk in hospitalized Iranian children. Acta Paediatr. 2012;101:e446-51.

8. Vazquez Martinez JL, Martinez-Romillo PD, Diez Sebastian J, Ruza Tarrio F. Predicted versus measured energy expenditure by continuous, online indirect calorimetry in ventilated, critically ill children during the early postinjury period. Pediatr Crit Care Med. 2004;5:19-27.

9. Verhoeven JJ, Hazelzet JA, van der Voort E, Joosten KF. Comparison of measured and predicted energy expenditure in mechanically ventilated children. Intensive Care Med. 1998;24:464-8.

10. White MS, Shepherd RW, McEniery JA. Energy expenditure in 100 ventilated, critically ill children: improving the accuracy of predictive equations. Crit Care Med. 2000;28:2307-12.

11. Mtaweh H, Smith R, Kochanek PM, Wisniewski SR, Fabio A, Vavilala MS, et al. Energy expenditure in children after severe traumatic brain injury. Pediatr Crit Care Med. 2014;15:242-9.

12. Coss-Bu JA, Jefferson LS, Walding D, David Y, Smith EO, Klish WJ, et al. Resting energy expenditure and nitrogen balance in critically ill pediatric patients on mechanical ventilation. Nutrition. 1998;14:649-52.

13. de Klerk G, Hop WC, de Hoog M, Joosten KF. Serial measurements of energy expenditure in critically ill children: useful in optimizing nutritional therapy? Intensive Care Med. 2002;28:1781-5.

14. Joosten KF, Verhoeven JJ, Hazelzet JA. Energy expenditure and substrate utilization in mechanically ventilated children. Nutrition. 1999;15:444-8.

15. Coss-Bu JA, Jefferson LS, Walding D, David Y, Smith EO, Klish WJ, et al. Resting energy expenditure in children in a pediatric intensive care unit: comparison of Harris-Benedict and Talbot predictions with indirect calorimetry values. Am J Clin Nutr. 1998;67:74-80.

16. Hong L, Shen LY, Zhao L. Resting energy expenditure in critically ill children with mechanical ventilation. Chin J Clin Nutr. 2015;23:1-7 (in Chinese).

17. Oosterveld MJ, Van Der Kuip M, De Meer K, De Greef HJ, Gemke RJ. Energy expenditure and balance following pediatric intensive care unit admission: a longitudinal study of critically ill children. Pediatr Crit Care Med. 2006;7:147-53. 
18. Mehta NM, Bechard LJ, Leavitt K, Duggan C. Cumulative energy imbalance in the pediatric intensive care unit: role of targeted indirect calorimetry. JPEN J Parenter Enteral Nutr. 2009;33:336-44.

19. White MS, Shepherd RW, McEniery JA. Energy expenditure measurements in ventilated critically ill children: withinand between-day variability. JPEN J Parenter Enteral Nutr. 1999;23:300-4.

20. Framson CM, LeLeiko NS, Dallal GE, Roubenoff R, Snelling LK, Dwyer JT. Energy expenditure in critically ill children. Pediatr Crit Care Med. 2007;8:264-7.

21. Gurgueira GL, Leite HP, Taddei JA, de Carvalho WB. Outcomes in a pediatric intensive care unit before and after the implementation of a nutrition support team. J Parenter Enteral Nutr. $2005 ; 29: 176-85$

22. Mikhailov TA, Gertz SJ, Kuhn EM, Scanlon MC, Rice TB, Goday PS. Early enteral nutrition is associated with lower mortality in critically ill children. JPEN J Parenter Enteral Nutr. 2014;38:459-66.

23. King W, Petrillo T, Pettignano R. Enteral nutrition and cardiovascular medications in the pediatric intensive care unit. JPEN J Parenter Enteral Nutr. 2004;28:334-8.

24. Mehta NM, Bechard LJ, Zurakowski D, Duggan CP, Heyland DK. Adequate enteral protein intake is inversely associated with 60-day mortality in critically ill children: a multicenter, prospective, cohort study. Am J Clin Nutr. 2015;102:199-206.

25. Mehta NM, Bechard LJ, Cahill N, Wang M, Day A, Duggan $\mathrm{CP}$, et al. Nutritional practices and their relationship to clinical outcomes in critically ill children-an international multicenter cohort study. Crit Care Med. 2012;40:2204-11.

26. Jotterand Chaparro C, Laure Depeyre J, Longchamp D, Perez MH, Taffé P, Cotting J. How much protein and energy are needed to equilibrate nitrogen and energy balances in ventilated critically ill children? Clin Nutr. 2016;35:460-7.

27. Vidigal MV, Leite HP, Nogueira PC. Factors associated with peptide-based formula prescription in a pediatric intensive care unit. J Pediatr Gastroenterol Nutr. 2012;54:620-3.

28. de Betue CT, van Waardenburg DA, Deutz NE, van Eijk HM, van Goudoever JB, Luiking YC, et al. Increased protein-energy intake promotes anabolism in critically ill infants with viral bronchiolitis: a double-blind randomised controlled trial. Arch Dis Child. 2011;96:817-22

29. van Waardenburg DA, de Betue CT, Goudoever JB, Zimmermann LJ, Joosten KF. Critically ill infants benefit from early administration of protein and energy enriched formula: a randomized controlled trial. Clin Nutr. 2009;28:249-55.

30. Meert KL, Daphtary KM, Metheny NA. Gastric vs. small-bowel feeding in critically ill children receiving mechanical ventilation: a randomized controlled trial. Chest. 2004;126:872-8.

31. Kamat P, Favaloro-Sabatier J, Rogers K, Stockwell JA. Use of methylene blue spectrophotometry to detect subclinical aspiration in enterally fed intubated pediatric patients. Pediatr Crit Care Med. 2008;9:299-303.

32. Snmez Düzkaya D, Yildiz S. Effect of two different feeding methods on preventing ventilator associated pneumonia in the paediatric intensive care unit (PICU): a randomised controlled study. Aust Crit Care. 2016;29:139-45.

33. Mehta NM, McAleer D, Hamilton S, Naples E, Leavitt K, Mitchell $\mathrm{P}$, et al. Challenges to optimal enteral nutrition in a multidisciplinary pediatric intensive care unit. JPEN J Parenter Enteral Nutr. 2010;34:38-45.

34. Canarie MF, Barry S, Carroll CL, Hassinger A, Kandil S, Li S, et al. Risk factors for delayed enteral nutrition in critically ill children. Pediatr Crit Care Med. 2015;16:e283-9.

35. Lambe C, Hubert P, Jouvet $\mathrm{P}$, Cosnes J, Colomb V. A nutritional support team in the pediatric intensive care unit: changes and factors impeding appropriate nutrition. Clin Nutr. 2007;26:355-63.
36. Briassoulis G, Filippou O, Kanariou M, Hatzis T. Comparative effects of early randomized immune or non-immune-enhancing enteral nutrition on cytokine production in children with septic shock. Intensive Care Med. 2005;31:851-8.

37. Briassoulis G, Filippou O, Hatzi E, Papassotiriou I, Hatzis T. Early enteral administration of immunonutrition in critically ill children: results of a blinded randomized controlled clinical trial. Nutrition. 2005;21:799-807.

38. Jacobs BR, Nadkarni V, Goldstein B, Checchia P, Ayad O, Bean J, et al. Nutritional immunomodulation in critically ill children with acute lung injury: feasibility and impact on circulating biomarkers. Pediatr Crit Care Med. 2013;14:e45-e56.

39. Briassoulis G, Filippou O, Kanariou M, Papassotiriou I, Hatzis T. Temporal nutritional and inflammatory changes in children with severe head injury fed a regular or an immune-enhancing diet: a randomized, controlled trial. Pediatr Crit Care Med. 2006;7:56-62.

40. Carcillo JA, Dean JM, Holubkov R, Berger J, Meert KL, Anand $\mathrm{KJ}$, et al. The randomized comparative pediatric critical illness stress-induced immune suppression (CRISIS) prevention trial. Pediatr Crit Care Med. 2012;13:165-73.

41. Al-Biltagi MA, Abo-Elezz AA, Abd-Elhafez MA, Mabrouk MM, Suliman GA. Beneficial effects of omega-3 supplement to the enteral feeding in children with mild to moderate sepsis. J Intensive Care Med. 2017;32:212-7.

42. Wang Y, Zhang J, Li X, et al. Effect of early enteral nutrition with polyunsaturated fatty acids $(\omega 3)$ on intestinal barrier function in children with severe acute pancreatitis. Chin Pediatr Emerg Med. 2014;21:520-2.

43. Hu Y, Jiang ZF. Title of the chapter?? In: Zhu FT, editor. Zhu Futang textbook of pediatrics, 8th ed. Beijing: People's Health Publishing House; 2015. p. 2756.

44. Fivez T, Kerklaan D, Mesotten D, Verbruggen S, Wouters PJ, Vanhorebeek I, et al. Early versus late parenteral nutrition in critically ill children. N Engl J Med. 2016;374:1111-22.

45. Jordan I, Balaguer M, Esteban ME, Cambra FJ, Felipe A, Hernández L, et al. Glutamine effects on heat shock protein 70 and interleukines 6 and 10: randomized trial of glutamine supplementation versus standard parenteral nutrition in critically ill children. Clin Nutr. 2016;35:34-40.

46. Ramos-Silva V, Castello JS, da Motta LL, Pereira FE, Oliveira NS, Lamounier JA, et al. Pulmonary lesions and total parenteral nutrition in children admitted to a pediatric intensive care unit. J Pediatr (Rio J). 2005;81:155-61.

47. Kyle UG, Jaimon N, Coss-Bu JA. Nutrition support in critically ill children: underdelivery of energy and protein compared with current recommendations. J Acad Nutr Diet. 2012;112:1987-92.

48. de Oliveira Iglesias SB, Leite HP, Santana e Meneses JF, de Carvalho WB. Enteral nutrition in critically ill children: are prescription and delivery according to their energy requirements? Nutr Clin Pract. 2007;22:233-9.

49. Wakeham M, Christensen M, Manzi J, Kuhn EM, Scanlon M, Goday PS, et al. Registered dietitians making a difference: early medical record documentation of estimated energy requirement in critically ill children is associated with higher daily energy intake and with use of the enteral route. J Acad Nutr Diet. 2013;113:1311-6.

50. Meyer R, Harrison S, Sargent S, Ramnarayan P, Habibi P, Labadarios D. The impact of enteral feeding protocols on nutritional support in critically ill children. J Hum Nutr Diet. 2009;22:428-36.

51. Petrillo-Albarano T, Pettignano R, Asfaw M, Easley K. Use of a feeding protocol to improve nutritional support through early, aggressive, enteral nutrition in the pediatric intensive care unit. Pediatr Crit Care Med. 2006;7:340-4. 